\title{
AVALIAÇÃO E AUTOPERCEÇÃO ANTROPOMÉTRICA DE CRIANÇAS DO 1. CICLO DO ENSINO BÁSICO DE VALONGO
}

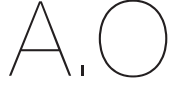

ARTIGO ORIGINAL

1 ITAU - Instituto Técnico de Alimentação Humana, S.A.

Rua das Cardosas, n. ${ }^{\circ} 1495$ 4425-510 S. Pedro Fins -

Maia, Portugal

${ }^{2}$ Câmara Municipal de

Valongo,

Avenida 5 de Outubro,

n. ${ }^{\circ} 160$,

4440-503 Valongo,

Portugal

${ }^{3}$ Faculdade de Ciências da Nutrição e Alimentação da Universidade do Porto, Rua Dr. Roberto Frias, 4200-465 Porto, Portugal

Endereço para correspondência António Teixeira Rua das Cardosas, n. ${ }^{\circ} 1495$ 4425-510 S. Pedro Fins - Maia, Portugal

antonio.teixeir@@trivalorpt

Histórico do artigo:

Recebido a 6 de junho de 2019 Aceite a 8 de outubro de 2019

\author{
ANTHROPOMETRIC ASSESSMENT AND SELF-PERCEPTION \\ AMONG PRIMARY SCHOOLCHILDREN IN VALONGO
}

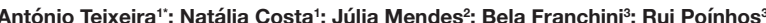

RESUMO

O excesso de peso em crianças é um grave problema de saúde pública, com repercussões na saúde e qualidade de vida no imediato e a longo prazo. Foram objetivos deste estudo: caraterizar crianças a frequentar o $1 .^{\circ}$ ciclo do ensino básico em termos de antropometria e imagem corporal (atual e desejada), avaliar a satisfação com a imagem corporal e relacionar o estado ponderal das crianças com a perceção e satisfação face à imagem corporal.

Para este estudo epidemiológico transversal foi selecionada uma amostra representativa da população estudantil do $1 .^{\circ}$ ciclo do ensino básico das escolas do Município de Valongo. Foi feita uma avaliação antropométrica (peso e estatura) e da perceção da imagem corporal (atual e desejada).

Das 481 crianças cujos dados foram analisados (52,2\% do sexo feminino) frequentavam o $1 .^{\circ}, 2 .^{\circ}, 3 .^{\circ}$ e $4 .^{\circ}$ ano, respetivamente, 112 , 123, 139 e 107 crianças. A proporção de crianças com excesso de peso (sobrepeso ou obesidade) foi de 41,0\% nas raparigas e $35,7 \%$ nos rapazes. Cerca de sete em cada dez crianças desejavam uma imagem diferente da atual, uma em cada oito desejava uma imagem correspondente a magreza e crianças com índice de massa corporal mais elevado indicavam imagens desejadas mais baixas. Os resultados reforçam a necessidade de políticas alimentares e intervenções que promovam simultaneamente uma correta evolução estaturo-ponderal e uma perceção adequada da imagem corporal, considerando as caraterísticas pessoais e contextuais dos indivíduos e grupos a que se destinam.

\section{PALAVRAS-CHAVE}

Alunos do $1 .{ }^{\circ}$ ciclo, Autoperceção, Avaliação antropométrica, Imagem corporal, Satisfação

ABSTRACT

Overweight among children is a serious public health problem, with repercussions in health and quality of life, both in the present and long term. The aims of this study were to characterize primary schoolchildren regarding anthropometry and body image (current and desired), to evaluate satisfaction with body image, and to relate children's weight status with their body image perception and satisfaction. For this cross-sectional study a representative sample of primary schoolchildren in the municipality of Valongo was selected. Anthropometric (weight and height) and body image (current and desired) assessments were made.

From a total of 481 children whose data were analyzed (52.2\% females), respectively $112,123,139$ and 107 attended the first, second, third and fourth school years. The proportion of overweight or obese children was $41.0 \%$ among girls and $35.7 \%$ among boys. About seven out of ten children desired a body image different from their current one, one out of eight desired an image corresponding to low weight, and children with higher body mass index selected lower desired images.

The results highlight the need for food policies and interventions promoting simultaneously an healthy weight and height progression and an adequate body image perception, taking into account the personal and contextual features of the target individuals and groups.

KEYWORDS

Schoolchildren, Self-perception, Anthropometric assessment, Body image, Satisfaction

\section{INTRODUÇÃo}

Desde há décadas a comunidade científica reconhece o excesso de peso como um grave problema de saúde pública, sendo crescente a importância atribuída ao excesso de peso em crianças $(1,2)$. Na Europa foram reportadas prevalências de excesso de peso em crianças dos 6 aos 9 anos de idade entre $18,4 \%$ e $42,5 \%$ em raparigas e entre 19,3\% e 49,0\% em rapazes; mesmo quando considerados apenas os casos de obesidade, os valores são igualmente alarmantes, chegando aos 17,3\% nas raparigas e 26,6\% nos rapazes $(3,4)$. A estes dados acresce que a prevalência de excesso de peso em crianças tem vindo a aumentar $(1,4)$, e na Europa verifica-se um gradiente norte-sul nas prevalências referidas, sendo as maiores prevalências registadas nos países do sul $(3,4)$. Em Portugal, entre 2008 e 2016, verificou-se uma redução de 37,9\% para 30,7\% na prevalência de excesso de peso (incluindo obesidade) e de $15,3 \%$ para $11,7 \%$ na de obesidade. Os rapazes são 
quem apresenta maior prevalência de obesidade (12,6\%), sendo que as raparigas registam maior prevalência de excesso de peso (incluindo obesidade) (31,6\%) (5).

Para além do maior risco de excesso de peso e problemas associados na idade adulta (6), o excesso de peso infantil está associado a diversas comorbilidades ainda durante a infância e adolescência, como hipertensão arterial (7), diabetes tipo 2 (8), doenças cardiovasculares (9) e distúrbios de sono $(10,11)$, bem como a outros problemas como pior qualidade de vida e baixa autoestima (12).

Apesar de na Europa as prevalências de crianças com baixo peso, baixa estatura ou baixo Índice de Massa Corporal (IMC) para a idade serem muito mais baixas comparativamente às de excesso de peso (3), estas condições não deixam de ser graves e, paradoxalmente, estão associadas a maior risco de excesso de peso no futuro (1).

A imagem corporal e, particularmente, a insatisfação com a mesma, influencia o autoconceito, não só em adultos como também em crianças e adolescentes. Para além de baixa autoestima e autoconfiança, a insatisfação com a imagem corporal pode causar depressão, dificuldades nas interações sociais e alterações comportamentais em crianças (13-18). Adicionalmente, se por um lado a insatisfação com a imagem corporal na infância aumenta o risco de excesso de peso, o excesso de peso leva à insatisfação corporal, havendo assim uma potenciação das consequências negativas das duas condições (14,19-20).

Os dados referentes à prevalência do excesso de peso em crianças evidenciam a necessidade de intervenções que a reduzam, minimizando assim as suas consequências imediatas e futuras. A avaliação concomitante da perceção e insatisfação com a imagem corporal será útil no delineamento destas estratégias, dadas as relações reportadas.

\section{OBJETIVOS}

Caraterizar crianças a frequentar o $1 .^{\circ}$ ciclo do ensino básico sob o ponto de vista antropométrico; caraterizar as crianças quanto à sua imagem corporal atual e desejada; avaliar a satisfação com a imagem corporal; relacionar o estado ponderal das crianças com a sua imagem corporal; e relacionar o estado ponderal com a satisfação face à imagem corporal.

\section{METODOLOGIA}

Este estudo epidemiológico transversal foi desenvolvido no âmbito do Projeto "CRESCER+", da responsabilidade do Instituto Técnico de Alimentação Humana, S.A. (ITAU), juntamente com a Faculdade de Ciências da Nutrição e Alimentação da Universidade do Porto (FCNAUP) e em parceria com o Município de Valongo (MV). O estudo foi autorizado pela Comissão de Ética da Universidade do Porto, Comissão Nacional de Proteção de Dados e Direção-Geral da Educação.

\section{Amostra}

No ano letivo de 2017-2018 estavam inscritas/os 3147 alunas/os no 1. ${ }^{\circ}$ ciclo do ensino básico, no MV, distribuídas/os por 144 turmas. Foram selecionadas para participar no estudo 29 turmas, correspondendo a $20 \%$ do número total de turmas. A amostragem foi aleatória, estratificada por agrupamentos e ano de escolaridade.

De um total de 632 alunas/os inscritas/os (idades entre os 6 e os 11 anos), 122 não foram avaliadas/os (taxa de participação de 80,7\%) por não devolução do consentimento informado $(n=62)$, recusa pela/o encarregada/o de educação ( $n=38$ ) ou ausência no dia da avaliação ( $n=22$ ). Posteriormente, foram excluídas da análise 29 crianças por impossibilidade de recolha completa dos dados.

\section{Procedimentos e instrumentos}

O consentimento informado das/os encarregadas/os de educação foi obtido por escrito. O termo de consentimento incluía questões a serem respondidas pelas/os encarregadas/os de educação que autorizassem a participação. As avaliações decorreram entre os dias 23 de abril e 16 de junho de 2018, nas instalações dos próprios estabelecimentos de ensino.

As avaliações foram realizadas por quatro avaliadoras/es com formação académica superior em Ciências da Nutrição e que participaram numa sessão de treino e uniformização de procedimentos. A avaliação antropométrica consistiu na medição do peso e da estatura, tendo sido posteriormente calculado o IMC. O peso foi medido com uma balança Tanita BC-601 e registado em kg com uma precisão de 0,1 kg. A estatura foi medida com um estadiómetro Seca 213 e registada em cm com uma precisão de $0,1 \mathrm{~cm}$. As medições foram realizadas de acordo com metodologia internacionalmente reconhecida (21), tendo sido uniformizado como procedimento a ausência de calçado e a manutenção da menor roupa de corpo em uso possível (t-shirt, calções/calças). O peso, a estatura e o IMC foram transformados em valores padronizados (z-scores) e classificados de acordo com os critérios da Organização Mundial da Saúde (22-24).

A avaliação da imagem corporal foi feita através da aplicação da Escala de Silhuetas de Collins para crianças, onde é apresentando à criança um conjunto de sete imagens que representavam ou raparigas ou rapazes com a mesma estatura e por ordem crescente de peso (1 = magreza; 7 = obesidade) (25). A criança deveria identificar a imagem percecionada como atual ("imagem atual"; "Das seguintes imagens, qual aquela que mais se parece contigo?") e a sua "imagem desejada" (“Destas imagens, com qual te gostarias de parecer?").

\section{Análise de dados}

A conversão dos valores de peso, estatura e IMC para z-scores foi feita através da extensão ao programa Microsoft Excel "ImsGrowth" (26). A análise estatística foi realizada no programa IBM SPSS versão 25.0 para Windows. A estatística descritiva consistiu no cálculo de frequências absolutas (n) e relativas (\%) e de médias e desvios-padrão (DP). A normalidade das variáveis cardinais foi avaliada através dos coeficientes de assimetria e de achatamento, sendo que todas apresentavam distribuição próxima da normal; dada a baixa amplitude da escala, as variáveis correspondentes às imagens foram tratadas como ordinais (testes não paramétricos). Foi usado o teste $t$ de student para comparar médias de amostras independentes, e foram usados os testes de Mann-Whitney e de Wilcoxon, respetivamente, para comparar ordens médias de amostras independentes ou emparelhadas. Utilizou-se o coeficiente de correlação de Spearman $\left(r_{s}\right)$ para medir o grau de associação entre pares de variáveis. Rejeitou-se a hipótese nula quando o nível de significância crítico para a sua rejeição (p) era inferior a 0,05.

\section{RESULTADOS}

Das 481 crianças cujos dados foram analisados, 251 (52,2\%) eram do sexo feminino. Frequentavam o $1 .^{\circ}, 2 .^{\circ}, 3 .^{\circ}$ e $4 .^{\circ}$ ano, respetivamente, 112, 123, 139 e 107 crianças. Não se verificaram diferenças significativas entre as idades das crianças do sexo feminino e masculino, quer para a totalidade da amostra quer por ano (dados não apresentados). 


\section{Caraterização antropométrica}

Nas Tabelas 1 e 2, apresenta-se a caraterização antropométrica da amostra. Não se verificaram diferenças significativas entre sexos para os z-scores de peso-para-idade, estatura-para-idade ou IMC-para-idade (Tabela 1). A proporção total de crianças com baixo peso-para-idade e baixa estatura-para-idade é inferior a $1 \%$ e a de crianças com baixo IMC-para-idade é inferior a 2\%; apenas 12 crianças (2,5\%) se encontram em uma ou mais destas condições. Por outro lado, a proporção de crianças com excesso de peso (sobrepeso ou obesidade) atinge 41,0\% nas raparigas e $35,7 \%$ nos rapazes (Tabela 2 ).

\section{Imagem corporal}

Não foram encontradas diferenças significativas entre sexos relativamente à imagem atual ou à imagem desejada. É de salientar que aproximadamente uma em cada oito crianças de cada sexo indicou como imagem desejada a primeira, que corresponde a uma situação de magreza (Tabela 3).

A Tabela 4 mostra a relação entre a imagem indicada por cada criança como correspondendo à sua imagem atual e a que corresponde à que gostaria de ter (desejada). Para ambos os sexos a imagem preferida é, em média, inferior à atual. Apenas cerca de três em cada 10 crianças estão satisfeitas com a sua imagem corporal (imagem preferida $=$ imagem atual), sendo que mais de metade das raparigas e mais de $40 \%$ dos rapazes indicaram como desejada uma imagem inferior à atual.

\section{Avaliação antropométrica e imagem corporal}

$\mathrm{Na}$ Tabela 5, apresentam-se as associações da imagem atual, da imagem desejada e da diferença entre as duas imagens com os z-scores de IMC-para-idade. Valores de IMC mais elevados estão associados a uma perceção da imagem atual também mais elevada, bem como a uma maior discrepância entre a imagem desejada e a atual (crianças com IMC mais elevado preferem uma imagem mais inferior à atual), mas a uma imagem desejada inferior.

Mais de três quartos das crianças com excesso de peso indicaram uma imagem preferida inferior à atual. Mesmo entre as crianças normoponderais verifica-se uma elevada prevalência de insatisfação com a imagem corporal, com cerca de $40 \%$ das raparigas e de um quarto dos rapazes a preferir também imagens inferiores à atual $e$ uma em cada quatro raparigas e mais de $30 \%$ dos rapazes a indicar uma imagem desejada superior à atual. Salienta-se ainda que cerca de um sexto das crianças com excesso de peso indicou uma imagem desejada igual à atual (Tabela 6).

\section{Tabela 1}

Caraterização antropométrica

\begin{tabular}{|c|c|c|c|c|c|c|c|c|c|c|c|c|}
\hline & \multicolumn{4}{|c|}{$\begin{array}{c}\text { PESO-PARA-IDADE } \\
\text { Z-SCORES } \\
\end{array}$} & \multicolumn{4}{|c|}{$\begin{array}{c}\text { ESTATURA-PARA-IDADE } \\
\text { Z-SCORES } \\
\end{array}$} & \multicolumn{4}{|c|}{$\begin{array}{l}\text { IMC-PARA-IDADE } \\
\text { Z-SCORES } \\
\end{array}$} \\
\hline & $\begin{array}{c}\text { SEXO } \\
\text { FEMININO }\end{array}$ & $\begin{array}{c}\text { SEXO } \\
\text { MASCULINO }\end{array}$ & $\mathbf{p}^{*}$ & TOTAL & $\begin{array}{c}\text { SEXO } \\
\text { FEMININO }\end{array}$ & $\begin{array}{c}\text { SEXO } \\
\text { MASCULINO }\end{array}$ & $\mathbf{p}^{*}$ & TOTAL & $\begin{array}{c}\text { SEXO } \\
\text { FEMININO }\end{array}$ & $\begin{array}{c}\text { SEXO } \\
\text { MASCULINO }\end{array}$ & $\mathbf{p}^{*}$ & TOTAL \\
\hline Média & 0,71 & 0,59 & 0,300 & 0,65 & 0,25 & 0,30 & 0,630 & 0,28 & 0,73 & 0,56 & 0,164 & 0,65 \\
\hline DP & 1,22 & 1,23 & & 1,22 & 1,06 & 1,01 & & 1,03 & 1,22 & 1,34 & & 1,28 \\
\hline Mínimo & $-2,38$ & $-2,64$ & & $-2,64$ & $-2,27$ & $-2,37$ & & $-2,37$ & $-2,35$ & $-2,69$ & & $-2,69$ \\
\hline Máximo & 4,34 & 4,01 & & 4,34 & 4,47 & 3,53 & & 4,47 & 4,05 & 3,87 & & 4,05 \\
\hline
\end{tabular}

O tamanho amostral para o peso-para-idade corresponde ao número de crianças até aos 9 anos (sexo feminino: $n=222$; sexo masculino: $n=202$; total: $n=424$ ).

* Diferença entre sexos

\section{Tabela 2}

Caraterização antropométrica: classificação

\begin{tabular}{|c|c|c|c|}
\hline \multirow{2}{*}{ CLASSIFICAÇÃO } & SEXO FEMININO & SEXO MASCULINO & TOTAL \\
\hline & n (\%) & n (\%) & n (\%) \\
\hline Baixo peso-para-idade $(z<-2)$ & $1(0,5)$ & $2(1,0)$ & $3(0,7)$ \\
\hline Baixa estatura-para-idade $(z<-2)$ & $2(0,8)$ & $2(0,9)$ & $4(0,8)$ \\
\hline Baixo IMC-para-idade $(z<-2)$ & $3(1,2)$ & $5(2,2)$ & $8(1,7)$ \\
\hline Normoponderabilidade $(-2 \leq \mathrm{z} \leq 1)$ & $145(57,8)$ & $143(62,2)$ & $288(59,9)$ \\
\hline Sobrepeso $(1<z \leq 2)$ & $63(25,1)$ & $46(20,0)$ & $109(22,7)$ \\
\hline Obesidade $(z>2)$ & $40(15,9)$ & $36(15,7)$ & $76(15,8)$ \\
\hline
\end{tabular}

A proporção de crianças com baixo peso-para-idade foi calculado para o número de crianças com idade até aos 9 anos (sexo feminino: $n=222$; sexo masculino: $n=202$; total: $n=424)$.

\section{Tabela 3}

Imagem atual e imagem desejada

\begin{tabular}{|c|c|c|c|c|c|c|c|c|c|c|}
\hline & & & \multicolumn{7}{|c|}{ IMAGEM } & \multirow{2}{*}{$\mathbf{p}^{*}$} \\
\hline & & & 1 & 2 & 3 & 4 & 5 & 6 & 7 & \\
\hline \multirow{2}{*}{$\begin{array}{l}\text { Imagem } \\
\text { atual }\end{array}$} & Sexo feminino & n (\%) & $\begin{array}{c}15 \\
(6,0)\end{array}$ & $\begin{array}{c}19 \\
(7,6)\end{array}$ & $\begin{array}{c}35 \\
(13,9)\end{array}$ & $\begin{array}{c}99 \\
(39,4)\end{array}$ & $\begin{array}{c}71 \\
(28,3)\end{array}$ & $\begin{array}{c}11 \\
(4,4)\end{array}$ & $\begin{array}{c}1 \\
(0,4)\end{array}$ & \multirow{2}{*}{0,158} \\
\hline & Sexo masculino & n (\%) & $\begin{array}{c}10 \\
(4,3) \\
\end{array}$ & $\begin{array}{c}10 \\
(4,3)\end{array}$ & $\begin{array}{c}59 \\
(25,7)\end{array}$ & $\begin{array}{c}91 \\
(39,6)\end{array}$ & $\begin{array}{c}49 \\
(21,3) \\
\end{array}$ & $\begin{array}{c}10 \\
(4,3)\end{array}$ & $\begin{array}{c}1 \\
(0,4) \\
\end{array}$ & \\
\hline \multirow{2}{*}{$\begin{array}{l}\text { Imagem } \\
\text { desejada }\end{array}$} & Sexo feminino & n (\%) & $\begin{array}{c}32 \\
(12,7)\end{array}$ & $\begin{array}{c}39 \\
(15,5)\end{array}$ & $\begin{array}{c}59 \\
(23,5)\end{array}$ & $\begin{array}{c}94 \\
(37,5)\end{array}$ & $\begin{array}{c}24 \\
(9,6)\end{array}$ & $\begin{array}{c}2 \\
(0,8)\end{array}$ & $\begin{array}{c}1 \\
(0,4)\end{array}$ & \multirow{2}{*}{0,182} \\
\hline & Sexo masculino & n (\%) & $\begin{array}{c}28 \\
(12,2)\end{array}$ & $\begin{array}{c}25 \\
(10,9)\end{array}$ & $\begin{array}{c}58 \\
(25,2)\end{array}$ & $\begin{array}{c}82 \\
(35,7)\end{array}$ & $\begin{array}{c}36 \\
(15,7)\end{array}$ & $\begin{array}{c}0 \\
(0,0)\end{array}$ & $\begin{array}{c}1 \\
(0,4)\end{array}$ & \\
\hline
\end{tabular}


Tabela 4

Relação entre a imagem atual e a imagem desejada

\begin{tabular}{lcc} 
& $\begin{array}{c}\text { SEXO FEMININO } \\
\mathbf{n}(\%)\end{array}$ & $\begin{array}{c}\text { SEXO MASCULINO } \\
\mathbf{n}(\%)\end{array}$ \\
\hline Imagem preferida $<$ atual & $141(56,2)$ & $102(44,3)$ \\
\hline Imagem preferida $=$ atual & $69(27,5)$ & $77(33,5)$ \\
\hline Imagem preferida $>$ atual & $41(16,3)$ & $51(22,2)$ \\
\hline $\mathrm{p}^{*}$ & $<0,001$ & $<0,001$ \\
\hline
\end{tabular}

* Diferença entre imagem preferida e imagem atual
Tabela 5

Associação das imagens atual e desejada com o IMC-para-idade

\begin{tabular}{lcc} 
& $\begin{array}{c}\text { SEXO FEMININO } \\
r_{s}(p)\end{array}$ & $\begin{array}{c}\text { SEXO MASCULINO } \\
r_{s}(p)\end{array}$ \\
\hline Imagem atual & $0,480(<0,001)$ & $0,479(<0,001)$ \\
\hline Imagem desejada & $-0,127(0,045)$ & $-0,236(<0,001)$ \\
\hline $\begin{array}{l}\text { Imagem desejada - } \\
\text { imagem atual }\end{array}$ & $-0,490(<0,001)$ & $-0,576(<0,001)$ \\
\hline
\end{tabular}

Tabela 6

Associação das imagens atual e desejada com o IMC-para-idade

\begin{tabular}{|c|c|c|c|c|c|}
\hline & & & $\begin{array}{l}\text { IMAGEM PREFERIDA } \\
\text { < ATUAL }\end{array}$ & $\begin{array}{l}\text { IMAGEM PREFERIDA } \\
\text { = ATUAL }\end{array}$ & $\begin{array}{c}\text { IMAGEM PREFERIDA } \\
>\text { ATUAL }\end{array}$ \\
\hline \multirow{2}{*}{$\begin{array}{l}\text { Baixo IMC-para-idade } \\
(z<-2)\end{array}$} & Feminino & $\mathrm{n}(\%)$ & $1(33,3)$ & $1(33,3)$ & $1(33,3)$ \\
\hline & Masculino & $n(\%)$ & $1(20,0)$ & $1(20,0)$ & $3(60,0)$ \\
\hline \multirow{2}{*}{$\begin{array}{l}\text { Normoponderabilidade } \\
(-2 \leq z \leq 1)\end{array}$} & Feminino & $\mathrm{n}(\%)$ & $59(40,7)$ & $51(35,2)$ & $35(24,1)$ \\
\hline & Masculino & $n(\%)$ & $35(24,5)$ & $63(44,1)$ & $45(31,5)$ \\
\hline \multirow{2}{*}{$\begin{array}{l}\text { Excesso de peso } \\
(z>1)\end{array}$} & Feminino & $\mathrm{n}(\%)$ & $81(78,6)$ & $17(16,5)$ & $5(4,9)$ \\
\hline & Masculino & n (\%) & $66(80,5)$ & $13(15,9)$ & $3(3,7)$ \\
\hline
\end{tabular}

\section{DISCUSSÃO DOS RESULTADOS}

Este estudo permitiu caraterizar sob o ponto de vista antropométrico e em termos de imagem corporal as/os alunas/os do $1 .^{\circ}$ ciclo do ensino básico do MV. Apesar da limitação decorrente da não participação de todas as crianças selecionadas, foi possível analisar os dados de mais de três quartos destas, o que, conjugado com o tipo de amostragem, constitui um dos pontos fortes deste estudo.

O resultado mais preocupante em termos de avaliação antropométrica prende-se com a elevada proporção de crianças de ambos os sexos com sobrepeso ou obesidade, cujos 38,5\% superam em cerca de cinco pontos percentuais os 33,9\% registados na região norte de Portugal em 2016 (5). Tal como noutros estudos, as raparigas apresentaram maiores proporções quer de sobrepeso quer de obesidade (27). Por outro lado, a prevalência de baixo peso, baixa estatura e/ou baixo IMC para a idade é reduzida, pelo que as intervenções a esse nível deverão ser individualizadas. Já a elevada prevalência de excesso de peso faz com que possam ser definidas estratégias de prevenção e intervenção mais abrangentes, nomeadamente de âmbito escolar (26). Para avaliação da imagem corporal foi utilizada uma escala desenvolvida e testada especificamente para crianças, apesar de não se encontrar ainda validada especificamente para as crianças portuguesas (27). Sete em cada dez crianças não estão satisfeitas com a sua imagem, sendo que a maioria deseja uma inferior à atual. A insatisfação com a imagem é mais prevalente em crianças com excesso de peso, o que é consistente com os resultados de outros estudos $(28,29)$. As raparigas apresentaram prevalência superior de insatisfação com a imagem, consistentemente com o descrito noutros estudos. Esta maior prevalência pode ser influenciada pela maior tendência para modelos de magreza como padrão para o sexo feminino (27-30). Mais rapazes preferiam uma imagem corporal superior à atual, talvez em função do padrão de muscularidade incutido culturalmente (2830). A insatisfação com a imagem corporal pode ter repercussões em termos de qualidade de vida no presente, mas também ser promotora de complicações de saúde no futuro, nomeadamente perturbações da alimentação e do comportamento alimentar, pelo que deve ser alvo privilegiado de atenção, especialmente no intervalo de idades das crianças estudadas (27-29).

Alguns resultados encontrados indicam que muitas crianças não têm uma adequada perceção da sua imagem corporal, pelo que este aspeto deve ser incluído em futuras intervenções que visem promover uma maior satisfação com a imagem corporal. É de salientar que não se verifica uma correspondência exata entre o estado ponderal e a (in)satisfação com a imagem, devendo por isso ser explorada concomitantemente a perceção de normas sociais relativamente ao estado ponderal e à imagem corporal. Estas normas podem variar em função de caraterísticas sociodemográficas, influência de familiares, pares e meios de comunicação $(28,29,31)$, pelo que as intervenções devem ser específicas para os grupos-alvo.

\section{CONCLUSÕES}

Os resultados deste estudo reforçam a necessidade de políticas alimentares e intervenções que promovam uma correta evolução estaturo-ponderal em crianças do $1 .^{\circ}$ ciclo simultaneamente com a promoção de uma perceção adequada da imagem corporal. Estas ações devem ter em conta as caraterísticas pessoais e contextuais dos indivíduos e grupos a que se destinam.

\section{REFERÊNCIAS BIBLIOGRÁFICAS}

1. World Health Organization. Report of the commission on ending childhood obesity. Geneva: WHO. 2016.

2. de Onis M, Blössner M, Borgji E. Global prevalence and trends of overweight and obesity among preschool children. Am J Clin Nutr. 2010, 92(5):1257-64.

3. Wijnhoven TM, van Raaij JM, Spinelli A, et al. WHO European Childhood Obesity Surveillance Initiative 2008: Weight, height and body mass index in 6-9-year-old children. Pediatr Obes. 2013;8(2):79-97.

4. Wijnhoven TM, Van Raaij JM, Spinelli A, et al. WHO European Childhood Obesity Surveillance Initiative: Body mass index and level of overweight among 6-9-year-old children from school year 2007/2008 to school year 2009/2010. BMC Public Health. 2014;14(1):806.

5. Rito A, Sousa R, Mendes S, Graça P, WHO Childhood Obesity Surveillance Initiative. COSI Portugal 2016. Lisboa: Instituto Nacional de Saúde Doutor Ricardo Jorge. 2017. Disponível em: http://repositorio.insa.pt/bitstream/10400.18/4857/8/COSI\%202016_ V2_fev2018.pdf.

6. Singh AS, Mulder C, Twisk JWR, et al. Tracking of childhood overweight into adulthood: A systematic review of the literature. Obes Rev. 2008;9(5):474-88.

7. Halbach SM, Flynn J. Treatment of obesity-related hypertension in children and adolescents. Curr Hypertens Rep. 2013;15(3):224-31.

8. Reinehr T. Type 2 diabetes mellitus in children and adolescents. Adolesc Med State 
Art Rev. 2010;4(6):270-81.

9. Herouvi D, Karanasios E, Karayianni C, Karavanaki K. Cardiovascular disease in childhood: The role of obesity. Eur J Pediatr. 2013;172(6):721-32.

10. Cappuccio FP, Taggart FM, Kandala N-B, et al. Meta-analysis of short sleep duration and obesity in children and adults. Sleep. 2008;31(5):619-26.

11. Börnhorst C, Wijnhoven TM, Kunešová M, et al. WHO European Childhood Obesity Surveillance Initiative: Associations between sleep duration, screen time and food consumption frequencies. BMC Public Health. 2015;15(1):442.

12. Griffiths LJ, Parsons TJ, Hill AJ. Self-esteem and quality of life in obese children and adolescents: A systematic review. Int J Pediatr Obes. 2010;5(4):282-304.

13. McElhone S, Kearney JM, Giachetti I, et al. Body image perception in relation to recent weight changes and strategies for weight loss in a nationally representative sample in the European Union. Public Health Nutr. 1999; 2(1a):143-51.

14. Moon YI, Park HR, Koo HY, Kim HS. Effects of behavior modification on body image, depression, and body fat in obese Korean elementary school children. Yonsei Med J. 2004; 45(1):61-7.

15. Schur EA, Sanders M, Steiner H. Body dissatisfaction and dieting in young children. Int J Eat Disord. 2000; 27:74-82.

16. Stice E, Hayward C, Cameron RP, Killen JD, Taylor CB. Body image and eating disturbances predict onset of depression among female adolescents. J Abnorm Psychol. 2000;109:438-44.

17. Wills W, Backett-Milburn K, Gregory S, Lawton J. Young teenagers' perceptions of their own and others' bodies: A qualitative study of obese, overweight and 'normal' weight young people in Scotland. Soc Sci Med. 2006 Jan;62(2):396-406.

18. Young-Hyman D, Schlundt SD, Herman-Wenderoth L, Bozylinski K. Obesity appearance and psychosocial adaptation in young African American children. J Pediatr Psychol. 2003; 28(7):463-72.

19. Davison KK, Birch LL. Predictors of fat stereotypes among 9 year old girls and their parents. Obes Res. 2004; 12(1):86-94.

20. Myers A, Rosen JC. Obesity stigmatization and coping: relation to mental symptoms, body image and self-esteem. Int J Obesity. 1999; 23:221-30.

21. World Health Organization. WHO child growth standards: Training course on child growth assessment. Geneva: WHO. 2008.

22. de Onis M, Onyango AW, Borghi E, Siyam A, Nishida C, Siekmann J. Development of a WHO growth reference for school-aged children and adolescents. Bull World Health Organ. 2007;85:660-7.

23. de Onis M, Lobstein T. Defining obesity risk status in the general childhood population: Which cut-offs should we use? Int J Pediatr Obes. 2010;5:458-60.

24. World Health Organization. Global database on child growth and malnutrition. Disponível em: http://www.who.int/nutgrowthdb/about/introduction/en/index5.html. 25. Collins E. Body figure perceptions and preferences among preadolescent children. Int J Eat Disord. 1991;10(2):199-208.

26. Pan, H.; Cole, T. ImsGrowth, a Microsoft Excel add-in to access growth references based on the LMS method (Version 2.68). 2009. Disponível em: http://www. healthforallchildren.co.uk/.

27. Coelho EM, Padez C, Moreira P, Rosado V, Mourão-Carvalhal I. BMI and selfperceived body shape in Portuguese children. Rev Psicol Desp. 2013;22(2):371-6.

28. Ricciardelli LA, McCabe MP. Children's body image concerns and eating disturbance. Clin Psychol Rev. 2001;21(3):325-44.

29. Neves CM, Cipriani FM, Meireles JFF, Morgado FFDR, Ferreira MEC. Body image in childhood: an integrative literature review. Rev Paul Pediatr. 2017;35(3):331-9.

30. Alves D, Pinto M, Alves S, Mota A, Leirós V. Cultura e imagem corporal. Motricidade. 2009;5(1):1-20.

31. Thompson JK, Heinberg LJ, Altabe M, Tantleff-Dunn S. Exacting beauty: theory, assessment, and treatment of body image disturbance. Washington: American Psychological Association. 1999. 